\title{
The End-Systolic Pressure-Volume Relationship in the Newborn Lamb: Effects of Loading and Inotropic Interventions
}

\author{
DAVID F. TEITEL, R. KLAUTZ, P. STEENDIJK, E. T. VAN DER VELDE, \\ F. VAN BEL, AND J. BAAN \\ Clinical Physiology Laboratory of the Departments of Pediatrics and Cardiology, Leiden University Hospital, \\ Leiden, The Netherlands
}

\begin{abstract}
Indices of global systolic performance of the newborn left ventricle exceed those of the adult, despite isolated tissue studies showing immature contractile mechanisms. To evaluate contractility in situ, we investigated the end-systolic pressure-volume relationship (ESPVR) by the conductance technique in nine newborn lambs. After percutaneous placement of catheters, we generated ESPVR by inferior vena cava occlusion, aortic occlusion, and volume infusion in two control states, during three levels of dobutamine infusion, and after propranolol. We performed linear and nonlinear regression analyses of the end-systolic points and derived the slope $\left(E_{e s}\right)$ and volume at $14 \mathrm{kPa}$ pressure. We found that reliable ESPVR could be obtained in almost all inferior vena cava and aortic occlusions (50 of 51 in each), but in only 18 of 27 volume infusions. Overall, linear regressions adequately defined the ESPVR ( 75 of 102 were not statistically different than nonlinear regressions; of those different, the mean linear $R^{2}$ was $0.934 \pm 0.048$ ). By multiple regression analysis, neither $E_{\text {es }}$ nor volume at $14 \mathrm{kPa}$ significantly changed with dobutamine, but both changed after propranolol (23\% less than control and $54 \%$ greater, respectively), supporting previous studies showing a limited contractile reserve in the newborn secondary to high resting $\beta$-adrenergic tone. Neither $E_{e s}$ nor volume at $14 \mathrm{kPa}$ was different between control states. However, $\mathrm{E}_{\mathrm{es}}$ was $25 \%$ less steep when generated by inferior vena cava than by aortic occlusion. We conclude that the ESPVR can be generated reliably and reproducibly in the newborn lamb and is relatively linear and sensitive to changes in contractility, but that it is also sensitive to the technique of load intervention. (Pediatr Res 29: 473-482, 1991)
\end{abstract}

\section{Abbreviations}

ESV, end-systolic volume

EDV, end-diastolic volume

ESPVR, end-systolic pressure-volume relationship

$P V$, pressure-volume

IVC, inferior vena cava

$E_{e s}$, elastance at end-systole (slope of the ESPVR)

$V_{14}$, volume at $14 \mathrm{kPa}$ pressure from the ESPVR

Received October 11, 1990; accepted January 11, 1991

Correspondence and reprint requests: David F. Teitel, Box 0130 , University of California, San Francisco, San Francisco, CA 94143.

D.F.T. was the recipient of an NIH Senior International Fellowship for this project.
At birth, the newborn left ventricle must increase its output 3fold above fetal values $(1-3)$ to meet the increased oxygen demands of respiratory and thermoregulatory work $(4,5)$. Studies performed on the newborn heart in situ have shown indices of systolic performance markedly elevated relative to those of the adult $(6,7)$, whereas studies of isolated tissues have suggested that its contractile mechanisms are underdeveloped (8-10). Most of the studies in situ have used single beat indices of performance during the preejection or ejection phase, however, which are significantly load or heart rate dependent. To correlate cardiac performance in situ with the structure and function of isolated tissue, it is preferable to use descriptors of systolic performance that are relatively load insensitive, and thus more closely reflect the intrinsic properties of the contractile mechanisms $(11,12)$.

Evaluation of ventricular systolic performance in the PV plane has been extensively performed in the adult human and dog (1322). In the adult, the ESPVR has been shown to be relatively sensitive to changes in inotropic state $(15,17,19,23)$, but evidence of nonlinearity (24-28) and afterload sensitivity (13, $23,29,30$ ) has been found. It is uncertain whether such problems would be more or less important in the newborn.

In the newborn, we have previously used an index of maximal elastance using a chronically implanted M-mode echo transducer and a pressure-tip catheter, and found that there was a very limited contractile reserve in the newborn lamb, secondary to a high resting $\beta$-adrenergic state (5). This technique, however, requires chronic implantation, and estimates of volume are based on single minor axis dimension measurements. Other techniques, such as two-dimensional echocardiography and angiocardiography, either are sensitive to changes in ventricular shape or orientation, are relatively insensitive to the very small volume changes that would occur in the newborn heart during the generation of the ESPVR, or affect mechanical function. In addition, none offer online numerical data acquisition, and these methods are thus dependent on observer interpretation, which is particularly undesirable when dealing with the very small volumes (ESV are in the range of 3 to $6 \mathrm{~mL}$ in the newborn lamb) of the newborn heart.

In contrast, the conductance catheter affords an instantaneous and continuous analog signal that can be converted online to digital data and does not affect cardiac mechanics or require chronic implantation. In the adult, it has been extensively validated in situ in the dog and human (31-33) and in the isolated dog heart (34), and has been used to construct the ESPVR in both species $(13,14,26,29,35,36)$. In the newborn, we have recently validated the catheter in the piglet against biplane cineangiography (37). In nine newborn pigs, we measured ventricular volume in a variety of conditions over which ESV and EDV varied greatly (during baseline conditions, isoproterenol infusion, and phenylephrine infusion, and after hemorrhage, volume infusion, and propranolol injection). We performed 110 separate runs, and measured EDV and ESV for each. From these 220 
data points, we performed a multiple regression analysis as described in the Appendix: the dependent variable was the volume estimate by the conductance catheter and the independent variables were the cineangiographic volume and a set of dummy variables representing the individual pigs. We found an excellent correlation $\left(\mathrm{R}^{2}\right.$ of 0.934$)$, a coefficient for the cineangiographic volume near identity $\left(1.1 \pm 0.08 \mathrm{~mL} \cdot \mathrm{mL}^{-1}\right)$, indicating a similar change in conductance volume for a given change in cineangiographic volume, and an intercept of $17.9 \mathrm{~mL}$, which represents the "parallel" conductance of structures contiguous to the left ventricular cavity (see below).

We undertook the present study to determine the reliability and reproducibility of the ESPVR in the newborn lamb generated by preload (IVC occlusion and volume loading) and afterload (aortic occlusion) alterations, and the sensitivity of the ESPVR to $\beta$-adrenergic stimulation (using dobutamine) and inhibition (using propranolol). In addition, we determined the nonlinearity of the ESPVR, its sensitivity to the type of loading intervention by which it is generated, and the stability of the preparation. Because the observed phenomena of nonlinearity and afterload dependence have been related to and explained on the basis of changes in inotropic state $(13,25,28)$, the present study in the newborn lamb with a high resting $\beta$-adrenergic state is especially meaningful.

\section{MATERIALS AND METHODS}

Nine Flevoland lambs were studied between 3 and $9 \mathrm{~d}$ of age, using a study protocol approved by the Animal Research Committee of the University of Leiden, the Netherlands. After premedication with ketamine hydrochloride $\left(3 \mathrm{mg} \cdot \mathrm{kg}^{-1}\right.$ i.v.), general anesthesia was maintained using a continuous infusion of ketamine hydrochloride $\left(8-30 \mathrm{mg} \cdot \mathrm{kg}^{-1} \cdot \mathrm{h}^{-1}\right)$. The lamb was intubated and ventilated with oxygen and air using a pressureregulated ventilator adjusted to maintain arterial $\mathrm{PO}_{2}$ and $\mathrm{PCO}_{2}$ in the normal range throughout the study. Upon ventilation, pancuronium hydrochloride was administered i.v. $\left(0.2 \mathrm{mg} \cdot \mathrm{kg}^{-1}\right)$ for muscle relaxation. An i.v. infusion of $10 \%$ dextrose in $0.5 \mathrm{~N}$ $\mathrm{NaCl}$ solution was continued throughout the study at about 100 $\mathrm{mL} \cdot \mathrm{kg}^{-1} \cdot \mathrm{h}^{-1}$, occasionally supplemented with $\mathrm{NaHCO}_{3}$ to maintain a normal base deficit $\left(\leq 5 \mathrm{mmol} \cdot \mathrm{L}^{-1}\right)$.

Via percutaneous approaches, 6-7 F self-sealing sheaths were placed in both right and left femoral arteries and veins. Atrial septostomy catheters (5 F; American Edwards Laboratories, Irvine, CA) were advanced via a femoral artery and vein to the mid-thoracic aorta and the IVC-right atrial junction, respectively, under fluoroscopic guidance. A $5 \mathrm{~F}$ angiographic catheter (American Edwards Laboratories) was advanced via the other femoral vein to the main pulmonary artery, and a $6 \mathrm{~F}$, eight-electrode conductance catheter (Webster Laboratories, Baldwin Park, CA) was advanced via the other femoral artery to the apex of the left ventricle. Ideally, the first (most distal) electrode should be in the apex of the left ventricle, and the proximal electrode alone should be above the aortic valve (Fig. 1). Under fluoroscopic guidance, when the distal tip of the catheter was advanced to the apex of the ventricle, the proximal electrode (electrode 8 in Fig. 1) was above the aortic valve. However, occasionally the total interelectrode distance was too great, so that electrode 7 was also above the valve. This could easily be ascertained by observing paradoxical motion of the volume signal of that segment during systole and diastole. In these instances, this 5th segment was removed from the summation of the segmental volumes during the calculation of total volume. Via a small incision on the left side of the neck, a $5 \mathrm{~F}$ sheath was inserted into a carotid artery and a $5 \mathrm{~F}$ pressure-tip catheter (Millar Instruments, Houston, TX) was advanced to just below the aortic valve and positioned to obtain a good signal. Repositioning of the pressure-tip catheter, under fluoroscopic guidance, was occasionally necessary during the study to avoid endocardial contact and pressure distortion.

Study protocol. Studies were performed under parasympathetic

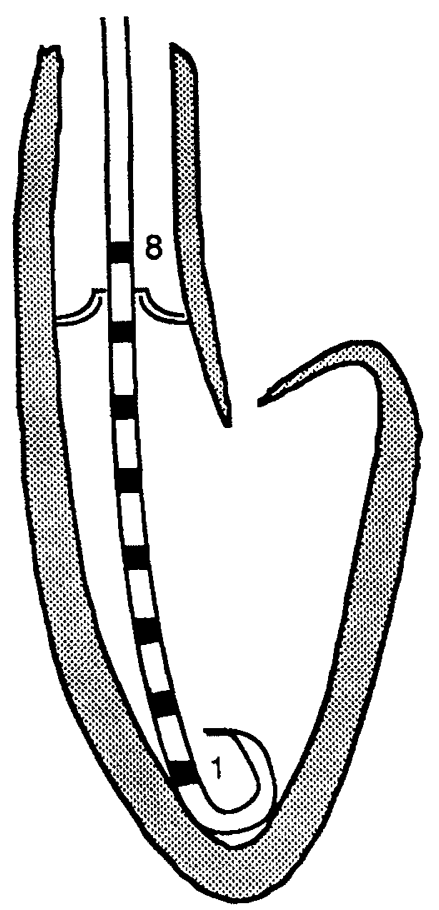

Fig. 1. Diagram of the conductance catheter. A $20-\mathrm{kHz}$ current is applied between electrode 1 , near the apex of the heart, and electrode 8 , above the aortic valve. Conductance of that current is measured between each pair of electrodes from electrode 2 through 7 . Thus, five segmental conductances are measured, from which segmental volumes are estimated. Total volume is calculated by summing these segmental volumes, ignoring the small apical volume of the ventricle.

blockade (atropine, $0.1 \mathrm{mg} \cdot \mathrm{kg}^{-1}$ i.v. PRN) to avoid reflex changes in heart rate induced by IVC or aortic occlusion. Instantaneous left ventricular pressure and volume were measured simultaneously using the pressure-tip and conductance catheters respectively and pressure-volume loops were constructed for each beat. During data acquisition, pressure and volume were changed by three loading techniques (IVC occlusion, aortic occlusion, and volume infusion), each performed under six conditions (two control periods, three levels of dobutamine infusion, and after propranolol administration). From these loops, ESPVR were generated for each run as described below. Each run was performed at end-expiration (the tracheal tube was not cuffed, and the ventilator was discontinued before data acquisition) after stabilization of pressure and volume. These brief periods of apnea were not associated with any changes in beat-to-beat heart rate, suggesting that stimulation of the chemoreceptor by either an increase in $\mathrm{PCO}_{2}$ or a decrease in $\mathrm{PO}_{2}$ is unlikely to have occurred.

In all nine lambs, ESPVR were obtained by IVC and aortic occlusion in each condition, the order of occlusion being randomized. The occlusions were performed by inflating the appropriate atrial septostomy balloon with $1.5 \mathrm{~mL}$ of $\mathrm{NaCl}$ solution over about $10 \mathrm{~s}$. In the first five lambs, ESPVR were also obtained during volume infusion after the IVC and aortic occlusions because of the possible late effects of volume infusion on contractility (38) [volume infusion was abandoned after analysis of the data of these first five lambs because of the unreliability of the obtained ESPVR (see Results)]. For the volume infusion runs, whole blood was infused through the pulmonary artery catheter. Up to $20 \mathrm{~mL} \cdot \mathrm{kg}^{-1}$ of blood was given over about $20 \mathrm{~s}$, and was discontinued early if stroke volume was seen to decrease appreciably. This happened in at least one half of the runs within the first $10 \mathrm{~mL} \cdot \mathrm{kg}^{-1}$ of the infusion. The first infusion in each lamb was with adult sheep blood. Immediately after the completion of each infusion, the same volume of blood was removed, which was then used as the infusate for the next infusion.

The first condition studied was the first control period (no 
drug administration), performed at least $30 \mathrm{~min}$ after completion of the surgical preparation and at least 15 min after hemodynamic stability (judged by a stable position of the PV loops). Thereafter, three levels of dobutamine were infused in a peripheral venous port of a sheath in a random order, at $4-5 \mu \mathrm{g} \cdot \mathrm{kg}^{-1}$. $\min ^{-1}$ (low level), at $8-10 \mu \mathrm{g} \cdot \mathrm{kg}^{-1} \cdot \mathrm{min}^{-1}$ (mid level), and at $16-$ $20 \mu \mathrm{g} \cdot \mathrm{kg}^{-1} \cdot \mathrm{min}^{-1}$ (high level). Stability of heart rate, arterial pressure, and stroke volume for at least $10 \mathrm{~min}$ was ensured before the acquisition of the ESPVR at each level of dobutamine infusion. Thirty min after the last dobutamine infusion was terminated, a second control period was studied. Thereafter, propranolol, $0.5 \mathrm{mg} \cdot \mathrm{kg}^{-1}$, was injected i.v., and ESPVR were again obtained after at least $15 \mathrm{~min}$ of hemodynamic stability. This order of study was chosen because we wished to separate the two control periods as much as possible, inasmuch as they were used to assess the stability of the preparation over time, and because it was necessary to administer the propranolol last, due to its prolonged effects.

All six conditions were studied in all nine lambs, except in the first lamb, in which only the first control, the mid level dobutamine infusion, and propranolol were studied. Thus, there were 51 ESPVR runs obtained during IVC occlusion, 51 during aortic occlusion, and 27 during volume infusion. In addition, we repeated the IVC and aortic runs in each condition in the last five lambs to determine the reproducibility of the ESPVR in the same condition obtained by the same loading technique. These second sets of occlusions were only used in the analysis of reproducibility.

Data acquisition and analysis. The fluid-filled sideport of an arterial cannula was connected to a pressure transducer and displayed on a paper recorder and screen for continuous monitoring. The pressure-tip catheter was connected via a conditioning amplifier to the recorder for pressure monitoring, and also displayed with the total volume signal from the conductance catheter on an X-Y oscilloscope for continuous monitoring of PV loops. Typical recorder tracings and PV loops are presented in Figures 2 and 3. The conductance catheter was connected to the Sigma-5 signal-conditioner-processor (Leycom, Oegstgeest, The Netherlands), which supplied the current, computed timevarying, segmental conductances, and generated analog output to the recorder and oscilloscope. An ECG was also generated from the conductance signals by the Sigma- 5 from two of the electrodes on the catheter. During each run of data acquisition, aortic and left ventricular pressure, ECG, and the five segmental conductance signals composing left ventricular volume were digitized with 12-bit accuracy on am IBM AT-compatible microcomputer, at a sampling rate of $200 \mathrm{~Hz}$, and saved on a hard disk for subsequent data analysis.

To convert measurements of segmental conductance, $G(i)$, to absolute segmental volumes $\mathrm{V}(\mathrm{i})$, the following formula is used:

$$
\mathrm{V}(\mathrm{i})=(1 / \alpha)\left(\mathrm{L}^{2} / \sigma_{\mathrm{b}}\right) \mathrm{G}(\mathrm{i})-\mathrm{V}_{\mathrm{c}}
$$

where $\mathrm{V}(\mathrm{i})$ is the segmental volume, $\alpha$ is a dimensionless slope constant, $\mathrm{L}$ is the interelectrode distance, $\sigma_{\mathrm{b}}$ is the specific conductivity of blood, and $V_{c}$ is a correction term caused by the conductance of structures surrounding the ventricular cavity (32). This "parallel" conductance $\left(\mathrm{V}_{c}\right)$ of structures contiguous to the left ventricular cavity was determined by injecting a very small amount $(0.1-0.2 \mathrm{~mL})$ of hypertonic $\mathrm{NaCl}$ solution into the pulmonary artery catheter during acquisition of the volume signal (32). The $\sigma_{\mathrm{b}}$ was measured in a $5-\mathrm{mL}$ cuvette connected to a model Sigma- 5 signal-conditioner-processor at the beginning of each condition and after each volume infusion. The conductivity of the blood used for the volume infusions was adjusted to match that of the lamb's blood before each run by the addition of small amounts of sterile water or hypertonic $\mathrm{NaCl}$ solution, as necessary. The slope constant, $\alpha$, is a correction factor for the difference between the estimate of a change in volume as calculated by the conductance catheter, as compared to a "true" change in volume as determined by another technique. For this study it was assumed to be 1 , although the average slope in the adult heart is about 0.8 (32). Calibration of and subsequent correction for $\alpha$ of each lamb was not necessary in this experiment; only within-lamb differences were considered in the statistical analyses because of the use of dummy variables representing animal-specific differences in the multiple regression analysis described in the Appendix. Thus, if any lamb had an $\alpha$ different than 1, this difference would be reflected in the coefficient of that lamb's dummy variable and would not have any effect on the statistical significance of the variables of interest.

Data analysis was performed using CONDUCT-PC (Leycom) on a microcomputer. The entire PV relationship was considered in terms of a time-varying elastance, and end-systole was defined as the point in each cardiac cycle of maximal elastance, i.e. at the maximum of the ratio

$$
\frac{P(t)}{V(t)-V_{d}}
$$

where $V_{d}$ is a correction term representing the unstressed, or "dead," volume of the ventricle. Because $V_{d}$ cannot be measured, it was determined for each run by an iterative approach. It was first assigned a value of 0 ; the initial end-systolic points for each loop were determined as maximal $\mathrm{P}(\mathrm{t}) / \mathrm{V}(\mathrm{t})$. A linear regression of end-systolic pressure versus ESV was performed, corrected for the randomness (mutual independence) of both $\mathrm{P}$ and $\mathrm{V}$ (39). From the resultant equation, a new $V_{d}$ was calculated, the endsystolic PV points were again determined, and the linear regression was again performed. This process was repeated until the difference between the last $V_{d}$ and the $V_{d}$ immediately before it was smaller than a preset error value of $0.1 \mathrm{~mL}$. At that point, the end-systolic PV points for each loop were saved in a file to be used in the subsequent analysis of the ESPVR for that run.

The ESPVR for each run was analyzed by performing simple linear and second order polynomial regression analyses on the ESPV points. From the linear ESPVR, two descriptors were calculated; the slope, or $E_{e s}$, and the $V_{14} . V_{14}$ was used rather than $V_{d}$ because the ESPVR has been demonstrated to be nonlinear to some extent in many preparations (24-28). Thus, extrapolation beyond the range of measurement is undesirable. $\mathrm{V}_{14}$ was specifically chosen because $14 \mathrm{kPa}$ was the overall mean starting pressure of all the runs in all lambs, so that pressure was most likely to be contained within the range of measurements whether pressure increased (aortic occlusion and volume loading) or decreased (IVC occlusion) during the run.

Statistical analyses were performed using StatView II (Abacus Concepts, Inc., Berkeley, CA) on a Macintosh IIx computer (Apple Computers, Cupertino, CA). Data are presented as mean \pm 1 SD unless otherwise specified. After regression analysis, an ESPVR was considered "reliable" if the correlation coefficient, $\mathrm{R}^{2}$, was greater or equal to 0.5 (so that at least one half of the total sum of squares is included within the regression sum of squares) and the $p$ value for the $t$ statistic of the slope (of the linear regression analysis) was less than or equal to 0.01 . To test whether ESPVR could be generated reproducibly using the same loading technique in the same condition, paired $t$ tests on both $E_{e s}$ and $V_{14}$ were performed, pairing the first aortic or IVC occlusion with the second occlusion performed on the same lamb in the same condition. Statistical techniques for determining the linearity of the ESPVR and its sensitivity to drug administration to loading technique and to the effects of time are complex and thus are presented in the Appendix.

Lastly, we determined whether parallel conductance changed relative to changes in ESV or EDV, as was found in the adult $\operatorname{dog}(40)$. We measured parallel conductance at the beginning of each condition, so that the starting volumes of all runs would be accurately corrected. We thus made 51 measurements of parallel conductance over a wide range of absolute volumes (ESV ranging from 0.59 to $5.38 \mathrm{~mL}$; EDV ranging from 3.23 to $10.25 \mathrm{~mL}$ ). The range of volumes was large within each lamb as well, because 

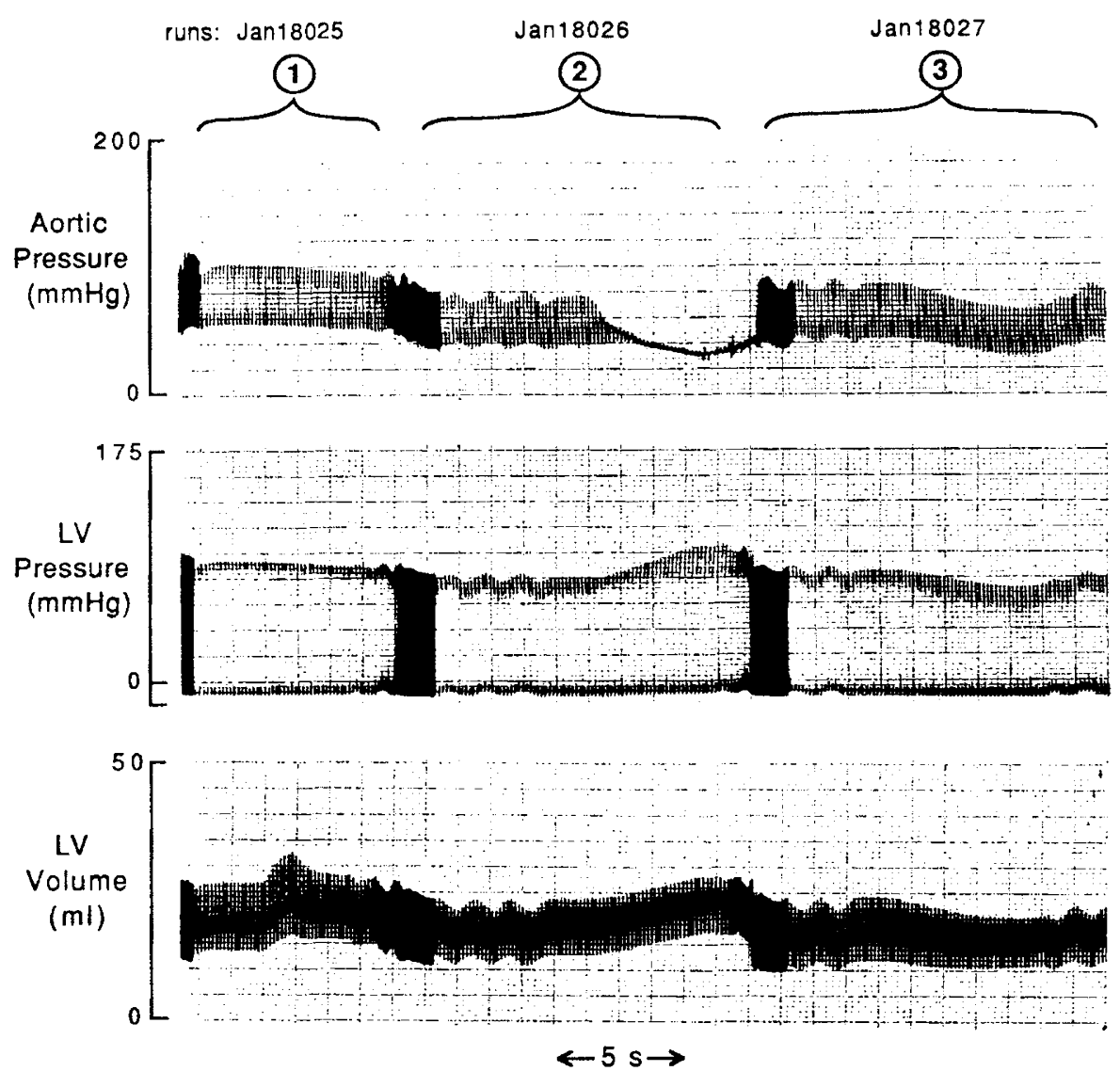

Fig. 2. Tracing from a typical set of three runs of data acquisition in one condition in one lamb. Run 1 is a determination of parallel conductance by the saline technique, in which there is a large increase in conductance-derived ventricular volume because of a change in blood conductivity, without a change in pressures. Run 2 is an aortic occlusion: the ventilator is discontinued, and after stabilization of pressures and volume, the aortic balloon is inflated; distal aortic pressure falls as left ventricular peak systolic pressure increases, and both ESV and EDV increase without a change in stroke volume. Run 3 is an IVC occlusion: after the ventilator is discontinued, the IVC balloon is inflated; aortic and left ventricular peak systolic pressures fall similarly, and ESV falls to a greater extent than EDV, as stroke volume decreases.

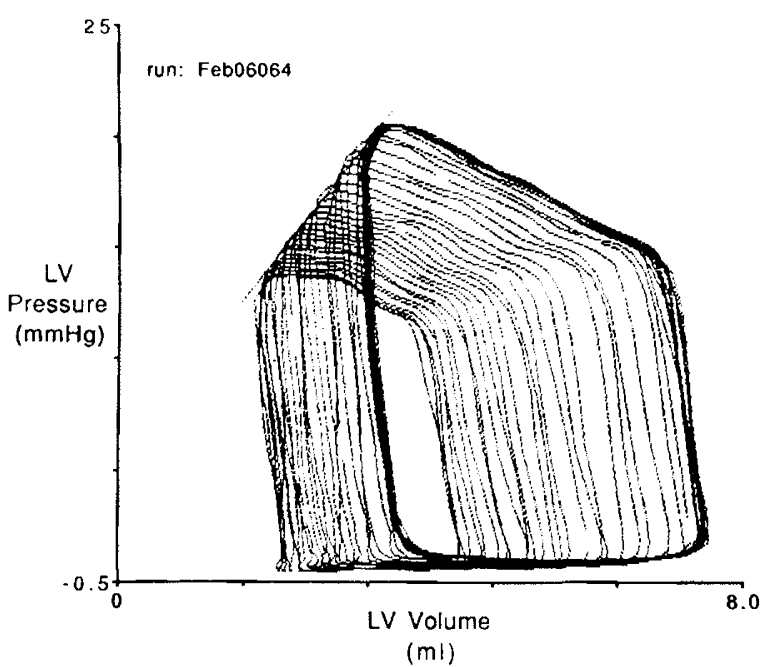

Fig. 3. Representative PV loops obtained during an IVC occlusion in one lamb.

the starting volumes after propanolol administration (ESV of 4.0 $\pm 0.7 \mathrm{~mL}$; EDV of $7.7 \pm 1.3 \mathrm{~mL}$ ) were much larger than those during control runs (ESV of $2.7 \pm 1.0 \mathrm{~mL}$; EDV of $6.7 \pm 1.6$ $\mathrm{mL}$ ), and the starting volumes during dobutamine infusion (ESV of $2.5 \pm 1.2 \mathrm{~mL} ; \mathrm{EDV}$ of $6.6 \pm 2.1 \mathrm{~mL}$ ) were slightly smaller. We analyzed the relationship between parallel conductance and ventricular volumes in the same manner as Boltwood et al. (40); we performed separate multiple regression analyses of parallel conductance against ESV and EDV, and included the set of dummy animal variables, $A_{1}$ to $A_{8}$, in each regression equation to separate the effects of the interanimal differences.

\section{RESULTS}

Reliability and linearity of ESPVR by different loading techniques. Table 1 summarizes the simple linear and second-order polynomial regressions of $\mathrm{P}$ versus $\mathrm{V}$ to generate ESPVR. By both IVC and aortic occlusion techniques, regression equations could be reliably fitted to the data in almost all cases ( 50 of 51 in both). The polynomial fit was significantly better than the linear fit in only nine of the 50 ESPVR generated by IVC occlusion, and in 18 of the 50 aortic occlusions. The overall mean correlation coefficients for the linear and polynomial regressions were very high and similar for the IVC and aortic occlusions (Table 1), and, in the 27 runs where polynomial fits were statistically better, the correlation coefficients for the linear regressions were still excellent and not significantly different from those of the polynomial regressions $(0.93 \pm 0.05$ versus $0.96 \pm$ 0.03 , respectively).

Conversely, regression fits for ESPVR generated by volume infusion could only be reliably made in 18 of the 27 runs. The overall mean correlation coefficient for those 18 runs was also lower than the coefficients for the IVC and aortic runs (Table 1). In addition, in six of the 18 runs, the polynomial fit was statistically better than the linear fit and the mean correlation coefficient of the polynomial fit of those six runs was also significantly higher $(0.96 \pm 0.03$ versus $0.89 \pm 0.7)$.

To further analyze why it was more difficult to obtain reliable 
Table 1. Results of linear and polynomial regression analyses of end-systolic PV points for each type of technique of generating ESPVR*

\begin{tabular}{lcccc}
\hline & Reliable/total & $\mathrm{R}^{2} /$ linear & $\mathrm{R}^{2} /$ polynomial & Polynomial $>$ linear \\
\hline IVC occlusion & $50 / 51$ & $0.91 \pm 0.12$ & $0.91 \pm 0.11$ \\
Aortic occlusion & $50 / 51$ & $0.89 \pm 0.10$ & $0.91 \pm 0.10$ \\
Volume infusion & $18 / 27$ & $0.84 \pm 0.13$ & $0.86 \pm 0.14$ & $18 / 50$ \\
$6 / 18$ & \\
\hline
\end{tabular}

* Reliable runs are those in which $\mathrm{R}^{2} \geq 0.50$ and $\mathrm{E}_{\mathrm{es}}>0, p \leq 0.01$. Polynomial fits are better than ( $>$ ) linear fits if the $t$ statistic of the second order term and the $F$ value comparing the two fits were significant, $p \leq 0.01$. Data are presented as mean $\pm \mathrm{SD}$.

ESPVR during volume infusion, we constructed cardiac function curves for the volume infusion runs. We speculated that rapid volume infusion in the newborn lamb, which has an extremely limited preload reserve $(41,42)$, may lead to overdistension of the left ventricle. If this were the case, the function curves would demonstrate a "descending limb," implying that contractility was acutely decreasing during the infusion. Data from a typical lamb is presented in Figure 4. The low-level and mid-level dobutamine infusions show normal cardiac function curves, shifted to the left of the control and propranolol curves. However, the high-level dobutamine infusion begins along the same curve as the lowand mid-level infusions, but quickly falls as stroke volume decreases with increasing EDV. The propranolol curve is, as expected, shifted well to the right, but also shows a prominent descending limb, and the control curve is very difficult to interpret. In total, six of the 18 "reliable" volume infusion runs show clear evidence of descending limbs, suggesting acute decreases in contractile state during the infusion. Because of these findings, we abandoned volume infusions in the last four lambs, and none of the subsequent statistical analyses uses the volume infusion data.

Reproducibility of ESPVR. We compared the indices of the linear regressions, $E_{\text {es }}$ and $V_{14}$, obtained during a first and second run of the same loading technique in the same condition. Fiftysix sets of duplicate runs (27 pairs of IVC occlusions and 29 pairs of aortic occlusions) were performed. There was no significant difference between the first and second runs for both $\mathrm{E}_{\mathrm{es}}(4.71 \pm$ 2.44 versus $4.68 \pm 2.49 \mathrm{kPa} / \mathrm{mL}, p>0.74)$ and $\mathrm{V}_{14}(2.89 \pm 1.28$ versus $2.84 \pm 1.24 \mathrm{~mL}, p>0.50$ ).

Sensitivity of ESPVR to drug infusion, loading technique, time lapse, and lambs. We performed multiple linear regression analyses for both $E_{\text {es }}$ and $V_{14}$ using dummy variables by effects coding, as described in the Appendix. The coefficients for the resultant regression equations are presented in Tables 2 and 3.

The overall mean $\mathrm{E}_{\text {es }}$ predicted by the regression analysis was $4.48 \mathrm{kPa} / \mathrm{mL}$. None of the coefficients representing the three levels of dobutamine infusion were statistically significant, indicating that the predicted $\mathrm{E}_{\mathrm{es}}$ at any level of dobutamine infusion was not different from the overall mean $\mathrm{E}_{\mathrm{es}}$, nor was it different from the predicted control $\mathrm{E}_{\mathrm{es}}$. Conversely, propranolol is predicted to decrease $\mathrm{E}_{\text {es }}$ to $3.54 \mathrm{kPa} / \mathrm{mL}(p \leq 0.01)$, which is a decrease of $21 \%$ versus the overall mean, or $23 \%$ versus the predicted control value. The time lapse coefficient was not significant, indicating that the preparation was stable with respect to $E_{\text {es }}$ between the two control conditions. The technique of generating the ESPVR was highly significant, with IVC occlusions being less steep and aortic occlusion steeper than the overall mean by $0.63 \mathrm{kPa} / \mathrm{mL}$. Thus, $\mathrm{E}_{\mathrm{es}}$ for IVC occlusions are predicted to be $25 \%$ less steep than aortic occlusions. There was a large amount of variability in $\mathrm{E}_{\text {es }}$ between lambs, as demonstrated by the large $F$ value for the set of lamb coefficients and a SD among the eight coefficients of $1.14 \mathrm{kPa} / \mathrm{mL}$. The ability of the regression equation to accurately predict $\mathrm{E}_{\mathrm{es}}$ in each condition and by each loading technique of occlusion is presented in Table 4 . It is apparent that the equation very closely predicts the measured values in each instance, which strongly suggests that no significant variable affecting $E_{e s}$ has been omitted from the regression analysis.

The predicted mean $\mathrm{V}_{14}$ was $2.78 \mathrm{~mL}$. The effects of dobutamine and propranolol on $V_{14}$ are similar to those seen on $E_{e s}$ : the coefficients representing the low and high levels of dobutamine infusion were not statistically significant, although the coefficient for the mid-level infusion was just significant at $p \leq$ 0.05 , and corresponded to a decrease (corresponding to a leftward shift of the ESPVR) of $0.41 \mathrm{~mL}$ from the overall mean value.

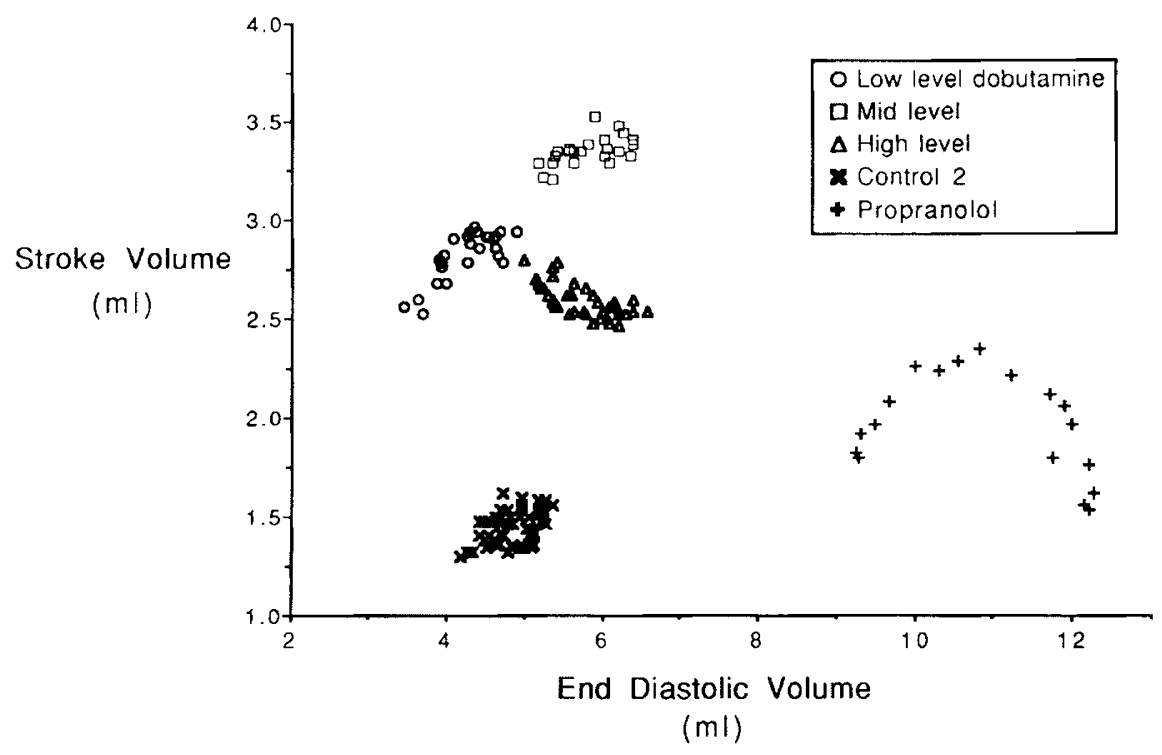

Fig. 4. Cardiac function curves in one lamb, generated during volume infusion in five conditions (the first control run was unsuccessful). Note that the propranolol curve is shifted down and to the right, consistent with a decrease in contractility, but that there is also a prominent descending limb suggesting that a further acute decrease in contractility occurred during the infusion. Also, although the high-level infusion of dobutamine begins along the same curve as the low and mid-level infusions, it shows a prominent descending limb as well. 
Table 2. $E_{e s}\left(k P a \cdot m L^{-1}\right):$ results of multiple linear regression analysis*

\begin{tabular}{|c|c|c|c|c|c|c|c|c|c|c|c|c|c|c|c|}
\hline \multirow[b]{2}{*}{ Variable } & \multirow[b]{2}{*}{$b_{0}$} & \multicolumn{4}{|c|}{ Drug interventions } & \multicolumn{2}{|c|}{ Time technique } & \multicolumn{8}{|c|}{ Between lambs } \\
\hline & & Dob/lo & $\mathrm{Dob} / \mathrm{mid}$ & Dob/hi & Prop & $\mathrm{C} 1 / \mathrm{C} 2$ & IVC/Ao & $\mathrm{L} 1$ & $\mathrm{~L} 2$ & L3 & $\mathrm{L} 4$ & L5 & L6 & L7 & L8 \\
\hline$b$ & 4.48 & 0.05 & 0.49 & 0.28 & -0.94 & 0.31 & -0.63 & 0.86 & -1.12 & -0.44 & -0.94 & 1.36 & -2.01 & 0.58 & -0.73 \\
\hline SEM & & 0.36 & 0.35 & 0.36 & 1.99 & 0.29 & 0.17 & 0.64 & 0.47 & 0.47 & 0.45 & 0.45 & 0.45 & 0.45 & 0.45 \\
\hline$p$ & & $>0.85$ & $>0.15$ & $>0.45$ & $<0.01 \dagger$ & $>0.25$ & $<0.001 \dagger$ & & & Coeffici & ents com & bined: & $<0.001 \dagger$ & & \\
\hline
\end{tabular}

* The coefficients $\mathrm{b}$ are in $\mathrm{kPa} \cdot \mathrm{mL}^{-1}$. $\mathrm{b}_{0}$, the intercept, or overall mean $\mathrm{E}_{\mathrm{es}}$. Variables: drug intervention variables are Dob/lo, assigned a value of +1 for low level infusion of dobutamine; Dob/mid, +1 for mid-level infusion of dobutamine; Dob/hi, +1 for high level infusion of dobutamine; and Prop, +1 for propranolol injection (all are assigned -1 for control runs); time lapse variable is $\mathrm{C} 1 / \mathrm{C} 2$, assigned +1 for first control and -1 for second; technique of occlusion variable is IVC/Ao, assigned +1 for IVC occlusion and -1 for aortic occlusion; lamb variables L1 through L8 represent the nine lambs. The regression equation was statistically significant $(p<0.0001)$. The SD of the between-lamb coefficients was $1.14 \mathrm{kPa}$. $\mathrm{mL}^{-1}$. The $p$ value is that of the corresponding $F$ test for that coefficient or group of coefficients.

$\uparrow$ Coefficient or group of coefficients has a significant effect on $E_{e s}$.

Table 3. $V_{14}(m L)$ : results of multiple linear regression analysis*

\begin{tabular}{|c|c|c|c|c|c|c|c|c|c|c|c|c|c|c|c|}
\hline & \multirow[b]{2}{*}{$\mathrm{b}_{0}$} & \multicolumn{4}{|c|}{ Drug interventions } & \multicolumn{2}{|c|}{ Time technique } & \multicolumn{8}{|c|}{ Between lambs } \\
\hline & & Dob/1o & $\mathrm{Dob} / \mathrm{mid}$ & Dob/hi & Prop & $\mathrm{Cl} / \mathrm{C} 2$ & IVC/Ao & $\mathrm{L} 1$ & L2 & $\mathrm{L} 3$ & $\mathrm{~L} 4$ & L5 & L6 & L7 & L8 \\
\hline $\mathrm{b}$ & 2.78 & -0.16 & -0.41 & -0.18 & 1.05 & -0.33 & -0.03 & -0.23 & -1.16 & 0.14 & -1.08 & -0.25 & 2.48 & 0.19 & 0.49 \\
\hline SEM & & 0.21 & 0.20 & 0.21 & 1.99 & 0.17 & 0.10 & 0.37 & 0.27 & 0.28 & 0.26 & 0.26 & 0.26 & 0.26 & 0.26 \\
\hline$p$ & & $>0.45$ & $<0.05 \dagger$ & $>0.40$ & $<0.0001 \dagger$ & $>0.06$ & $>0.75$ & \multicolumn{8}{|c|}{ Coefficients combined: $<0.001 \dagger$} \\
\hline
\end{tabular}

${ }^{*}$ The coefficients $\mathrm{b}$ are in $\mathrm{mL}$. See Table 2 for definition of variables. The regression equation was statistically significant $(p<0.0001)$. The SD of the between-lamb coefficients was $1.14 \mathrm{kPa} \cdot \mathrm{mL}^{-1}$. The $p$ value is that of the corresponding $F$ test for that coefficient or group of coefficients.

$\dagger$ Coefficient or group of coefficients has a significant effect on $V_{14}$.

Table 4. Values of $E_{\text {es }}\left(k P a \cdot m L^{-1}\right)$ and $V_{14}(m L)$ : comparisons between predicted and measured mean values overall, for each condition, and for each technique

\begin{tabular}{lccccc}
\hline & \multicolumn{2}{c}{$\mathrm{E}_{\text {es }}$} & & \multicolumn{2}{c}{$\mathrm{V}_{14}$} \\
\cline { 2 - 3 } \cline { 5 - 6 } \cline { 5 - 6 } & Predicted & Measured & & Predicted & Measured \\
\hline Overall & 4.48 & 4.48 & & 2.78 & 2.75 \\
Control 1 & 4.93 & 4.93 & & 2.15 & 2.15 \\
Control 2 & 4.31 & 4.32 & & 2.81 & 2.91 \\
Dobutamine/low & 4.54 & 4.43 & & 2.63 & 2.66 \\
Dobutamine/mid & 4.98 & 4.98 & & 2.38 & 2.38 \\
Dobutamine/high & 4.76 & 4.65 & & 2.61 & 2.64 \\
Propranolol & 3.52 & 3.51 & & 3.83 & 3.82 \\
IVC occlusions & 3.86 & 3.85 & & 2.76 & 2.75 \\
Aortic occlusions & 5.11 & 5.11 & & 2.81 & 2.75 \\
\hline
\end{tabular}

This is equivalent to a $15 \%$ leftward shift versus the overall mean but just a $7 \%$ shift from the predicted control value. The effect of propranolol was much greater, with a predicted increase (rightward shift) in $\mathrm{V}_{14}$ of $1.05 \mathrm{~mL}$, which is $38 \%$ greater than the overall mean and $54 \%$ greater than the predicted control value. There was no difference in $\mathrm{V}_{14}$ between the two control periods, nor between the two loading techniques of generating the ESPVR. The latter finding is an artifact of the index itself, however: $\mathrm{V}_{14}$ was chosen because $14 \mathrm{kPa}$ was the mean starting pressure over all the runs; thus, most of the IVC and aortic runs in a given condition begin at similar volumes near a pressure of $14 \mathrm{kPa}$. The ESPVR for the paired occlusions should thus "scissor" at about $14 \mathrm{kPa}$, as demonstrated in Figure 5. The interanimal variability was large, with a SD among the coefficients of $1.14 \mathrm{~mL}$. Lastly, as with $\mathrm{E}_{\mathrm{es}}$, the predicted values for $\mathrm{V}_{14}$ were very similar to the measured values (Table 4), indicating that no significant independent variable was omitted from the regression analysis.

Parallel conductance versus ESV and EDV. We performed multiple linear regression analyses for parallel conductance against ESV and EDV, using dummy lamb variables. The regression equations are presented in Tables 5 and 6 . The coefficients for ESV and EDV were very small, and neither approached statistical significance, indicating that there is no relationship between parallel conductance and either ventricular volume in our preparation.

\section{DISCUSSION}

Evaluation of left ventricular systolic performance in the PV plane during load alterations has given investigators a more specific tool to assess contractility in the in situ heart, because there appears to be less load dependency of such PV relationships than there is using single-beat preejection or ejection phase indices of performance $(14,18,19)$. The conductance catheter has been used in the adult dog and human simultaneously with micromanometer pressure measurements for the repeated and instantaneous display and acquisition of PV loops without affecting ventricular function $(13,26,29,36,43)$. PV loops have been analyzed by a variety of techniques $(13,14,35,44-46)$, but most commonly by generating ESPVR, to assess ventricular function in control conditions and in response to various hemodynamic perturbations. There have, however, been several concerns about the ESPVR, including those of nonlinearity both outside and within the range of measurement (24-28), load dependency, particularly related to the technique of generating the relationship itself $(13,15,23,29)$, and relative insensitivity to changes in contractility (43). We have recently performed studies validating the accuracy of the conductance catheter in the in situ newborn heart (37), and undertook the present study to critically analyze the ESPVR in the newborn. We found that we could reliably and reproducibly acquire ESPVR by both IVC and aortic occlusion but not by volume infusion, that nonlinearity is present but to a lesser extent than has recently been demonstrated in the adult heart (28), that the loading technique does affect the relationship, and that the preparation is stable throughout the $2 \mathrm{~h}$ of data acquisition.

Nonlinearity of the ESPVR would make any index derived from a linear regression analysis dependent upon the range over which the measurements were made. Thus, comparisons between subjects, or between states within a subject, become problematic. We do not attempt to make comparisons of absolute data between subjects (as discussed in the Appendix), but comparison of absolute data between states within a subject is a prerequisite to statistical analysis of the data. This nonlinearity may not be a major problem in the clinical evaluation of the patient in whom a global perspective of the PV loops is all that is required for therapeutic decisions (35), but it is a critical problem for experimental studies. It has become clear that the assumption of 

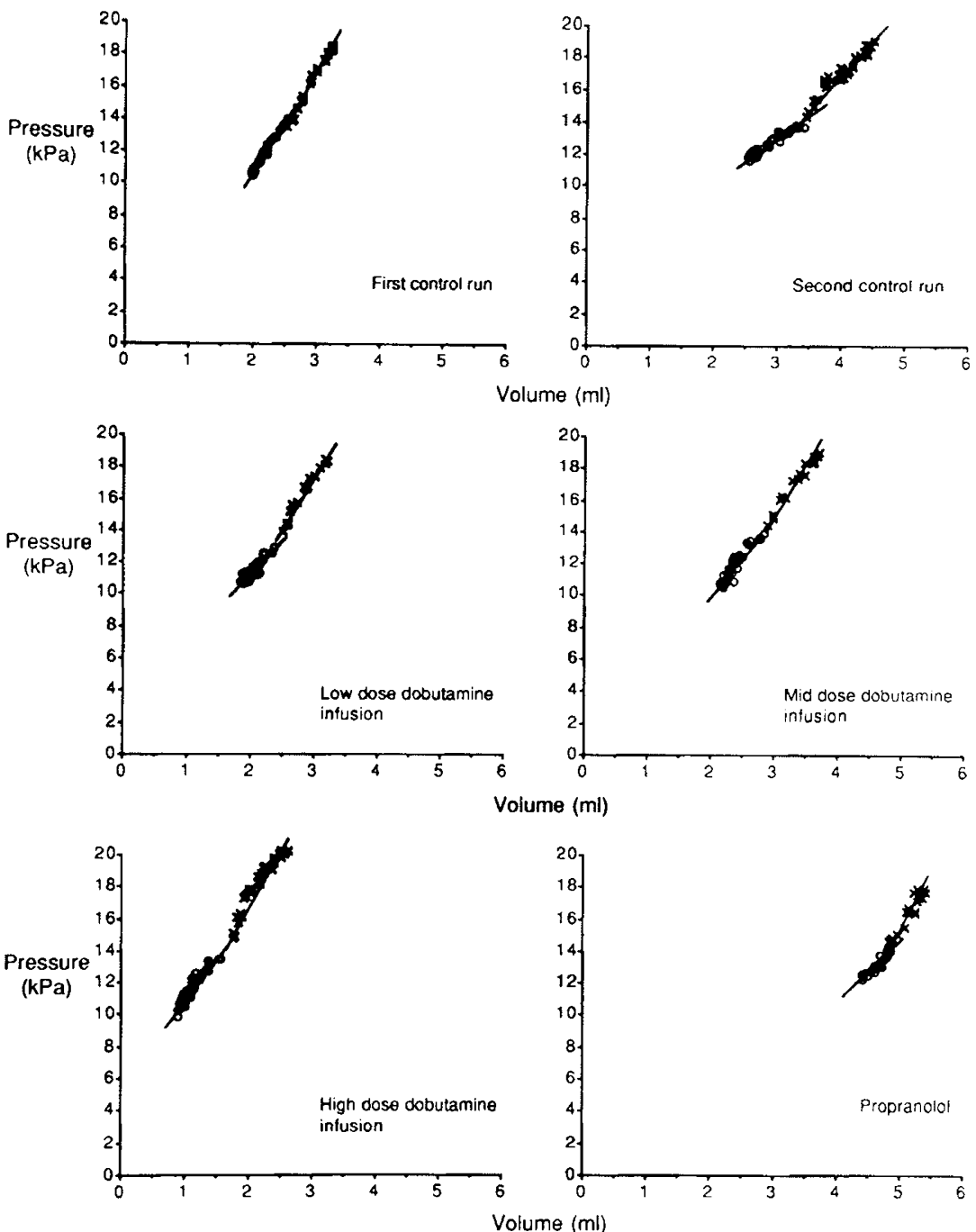

Fig. 5. Scissoring: the slope of the end-systolic PV points during aortic occlusions $(X)$ is steeper than that during IVC occlusions $(O)$ in all six conditions, and scissors at the starting pressure, approximately $14 \mathrm{kPa}$.

Table 5. Parallel conductance $(m L)$ : results of the multiple linear regression analysis vs ESV and interanimal variability (L1 through L8)*

\begin{tabular}{|c|c|c|c|c|c|c|c|c|c|c|}
\hline & \multirow[b]{2}{*}{$b_{0}$} & \multirow[b]{2}{*}{ ESV } & \multicolumn{8}{|c|}{ Between lambs } \\
\hline & & & $\mathrm{L} 1$ & $\mathrm{~L} 2$ & L3 & L4 & L5 & L6 & L7 & L8 \\
\hline $\mathrm{b}$ & 12.53 & -0.04 & 4.03 & -1.02 & -1.00 & 1.22 & -3.21 & 0.27 & -2.07 & 2.38 \\
\hline SEM & & 0.28 & 0.98 & 0.79 & 2.02 & 0.78 & 0.68 & 0.74 & 0.72 & 0.88 \\
\hline$p$ & & $>0.85$ & \multicolumn{8}{|c|}{ Coefficients combined: $<0.001 \dagger$} \\
\hline
\end{tabular}

${ }^{*} b$, coefficient for each variable. The regression equation was statistically significant $(p<0.0001)$. The $p$ value is that of the corresponding $F$ test for that coefficient or group of coefficients.

$\uparrow$ The group of coefficients has a significant effect on parallel conductance.

Table 6. Parallel conductance $(m L)$ : results of multiple linear regression analysis vs EDV and interanimal variability (L1 through L8)

\begin{tabular}{|c|c|c|c|c|c|c|c|c|c|c|}
\hline & \multirow[b]{2}{*}{$\mathrm{b}_{0}$} & \multirow[b]{2}{*}{ EDV } & \multicolumn{8}{|c|}{ Between lambs } \\
\hline & & & $\mathrm{Ll}$ & L2 & L3 & $\mathrm{L} 4$ & L5 & L6 & L7 & L8 \\
\hline $\mathrm{b}$ & 12.97 & -0.08 & 3.94 & -1.01 & -1.00 & 1.13 & -3.20 & 0.34 & -2.00 & 2.46 \\
\hline SEM & & 0.18 & 1.01 & 0.72 & 2.02 & 0.81 & 0.67 & 0.73 & 0.73 & 0.89 \\
\hline$p$ & & $>0.65$ & \multicolumn{8}{|c|}{ Coefficients combined: $<0.001 \uparrow$} \\
\hline
\end{tabular}

${ }^{*} \mathrm{~b}$, coefficient for each variable. The regression equation was statistically significant $(p<0.0001)$. The $p$ value is that of the corresponding $F$ test for that coefficient or group of coefficients.

$\dagger$ The group of coefficients has a significant effect on parallel conductance. 
linearity beyond the range of measurement is invalid, so that indices such as $V_{0}$ obtained by linear extrapolation of the data should not be used. In the current study, we used $V_{14}$ rather than $\mathrm{V}_{0}$ so that extrapolation was minimized.

However, significant nonlinearity of the ESPVR within the range of its generation has also been found in the adult heart recently (28), which would limit the usefulness of $V_{14}$ and $E_{\text {es. }}$. The theoretical basis for the linearity of the ESPVR is tenuous at best. One needs to assume that the isovolumic ESPVR is linear over the range in load of the ejecting ESPVR and that the ejecting beats always attain the same pressure as isovolumic beats of the same ESV [alternatively, there can be a fixed pressure deficit (47) or a balance between the opposing effects that tend to cause a pressure deficit and those that cause a pressure excess $(48,49)]$. Pressure deficits during ejection probably do occur to some extent, caused by shortening deactivation and the internal resistance of series elastic elements. Recent studies in the adult dog have shown nonlinearity in a variety of settings, including high contractile states $(25,26)$, over a wide range of preload $(27$, 28 ) and during balloon aortic occlusion (24). The nonlinearity was considered significant only in those studies performed over a high range in preload $(27,28)$, in which stroke volume changes greatly and thus quite variable pressure deficits can occur during the generation of the ESPVR. In contrast, we did not find significant nonlinearity, particularly when the ESPVR was generated by IVC occlusion. Moreover, when we found statistically significant nonlinearity, the linear regressions still fit the endsystole points to an excellent degree, with a mean correlation coefficient of 0.93 . Thus, we did not correct our indices of the ESPVR, $E_{\text {es }}$ and $V_{14}$, for nonlinearity in the multiple regression analysis. It is probable that nonlinearity was not a major finding in our study because the range of stroke volumes over which the ESPVR were generated was very small. Because the newborn lamb heart is very small (absolute diastolic volumes averaging about $6 \mathrm{~mL}$, and stroke volumes about $4 \mathrm{~mL}$ ) and beats quickly (averaging about 200-240 $\mathrm{min}^{-1}$ ), we were able to obtain many (on the average, about 40) PV loops within $10 \mathrm{~s}$ of data acquisition over a narrow range of volumes. Within these narrow confines of time and load, it is not surprising that substantial nonlinearity was not apparent.

Although we did not find substantial nonlinearity in our newborn preparation, we did find that the technique of generating the ESPVR significantly affected it. We could not produce reliable ESPVR with volume infusion, unlike in the adult dog (13). This was almost certainly related to the fact that, unlike the adult, the newborn has a very limited preload reserve $(41,42)$. In addition to a limited preload reserve, the newborn left ventricle is sensitive to modest increases in afterload that occur during volume infusion (50). Thus, increasing EDV by volume infusion may not be associated with an increase in stroke volume and may cause a decrease in contractile state. This would render the obtained ESPVR, which depends upon a constant contractile state, uninterpretable. The presence of a changing contractile state was evident from the prominent descending limbs from several cardiac function curves constructed during volume loading. Thus, volume loading is an inappropriate technique to construct ESPVR in the newborn.

IVC and aortic occlusion both generated reliable and reproducible ESPVR, but those generated by aortic occlusion were significantly steeper. We were unable to determine whether the position of the ESPVR was different between IVC and aortic occlusions because our positional index of the ESPVR, $\mathrm{V}_{14}$, was the starting pressure and volume of both occlusions. However, in the adult dog, ESPVR generated during aortic occlusion were steeper and also shifted to the left as compared with those generated during volume loading (13). For the same reasons that nonlinearity may exist, the ESPVR slope may be steeper during aortic occlusion. During both aortic occlusion and IVC occlusion, stroke volume decreases. This would lessen shortening deactivation, causing the ejecting beat to more closely approach the isovolumic PV line. This would cause the ESPVR generated as pressure was increasing (aortic occlusion) to be steeper than that generated as pressure was decreasing (IVC occlusion). However, internal resistance may be quite different at the different levels of arterial impedance caused by aortic and IVC occlusion because of substantially different pressures and velocities of ejection; this might have an opposite effect on the relative slopes of the ESPVR during aortic and IVC occlusion. Also, it is possible that contractility is directly affected by changes in arterial impedance. Baan and van der Velde (29) found that the slope of the maximum change in pressure per unit time-left ventricular EDV relationship was also steeper during aortic occlusion as compared to volume infusion. This preejection phase index derived from the PV plane cannot be affected by either shortening deactivation or internal resistance. The authors speculated that there was a direct increase in contractility secondary to an increase in arterial impedance, possibly related to changes in calcium turnover. We found a much smaller difference in the slope of the ESPVR generated by aortic versus IVC occlusion than they found when the ESPVR was generated by aortic occlusion versus volume loading. This may be explained by the fact that the resting contractile state of the newborn is much higher than that of the adult (5), so that the reserve to increase contractility further is limited.

Lastly, it is possible that at least some of the differences in $\mathrm{E}_{\mathrm{es}}$ generated by aortic and IVC occlusion may have been underestimated because of problems associated with the technique itself. Boltwood et al. (40) have found that parallel conductance volume increases with increases in ventricular volume. We measured parallel conductance at the beginning of each condition so that both the aortic and IVC occlusion had parallel conductance estimates performed at their starting volumes. However, ventricular volumes increase during the aortic occlusion but decrease during IVC occlusion. If parallel conductance were increasing simultaneous to increases in intraventricular volumes during aortic occlusion, $\mathrm{E}_{\mathrm{es}}$ would be overestimated during aortic occlusion; conversely, $E_{\text {es }}$ would be underestimated during IVC occlusion, which together would obscure the scissoring. However, contrary to the study of Boltwood et al. (40), we did not find a significant effect of either ESV or EDV on $\alpha \mathrm{V}_{\mathrm{c}}$. Although we do believe that it is important to measure parallel conductance at the beginning of any new condition because changes may indeed occur related to changes in left atrial or right ventricular volume or lung mechanics, it does not appear that we had a systematic error in the measurement of the absolute ventricular volumes related to acute changes in left ventricular volume in the absence of simultaneous corrections for parallel conductance.

The sensitivity of ESPVR to changes in contractility are critical to its use in the evaluation of ventricular performance, although its strength as an index of contractility is probably more related to its relative load insensitivity than to sensitivity to changes in contractility (14). In our newborn lamb preparation, we do not have a standard against which to compare the sensitivity of ESPVR relative to changes in contractility. However, it has been shown that the newborn lamb heart is functioning at a much greater level of performance than the adult sheep heart (6), and that it has a limited reserve to increase performance in response to volume loading $(41,42)$, or to digoxin administration (51). In addition, Baylen et al. (52) have shown that the newborn heart is functioning under a significant amount of adrenal catecholamine stimulation, and we have previously shown that it has a limited contractile reserve related to a high resting $\beta$-adrenergic state (5). Thus, we would expect little effect of dobutamine infusion on ESPVR, but a large effect of propranolol. This is indeed what we found. Although each level of dobutamine was associated with a slight increase in $\mathrm{E}_{\mathrm{es}}$ as compared to the overall mean value, none of the effects approached statistical significance. Conversely, propranolol decreased $\mathrm{E}_{\mathrm{es}}$ significantly. We found similar effects of dobutamine and propranolol on $V_{14}$. Each level of dobutamine slightly shifted the ESPVR to the left, 
although it was only significant (with a $p$ value of 0.0495 ) with the mid level of dobutamine, and only corresponded with a shift of $7 \%$ versus the predicted control value. Conversely, propranolol caused a large increase in $\mathrm{V}_{14}$, or rightward shift in the ESPVR, to a value that was $54 \%$ greater than the predicted control value. Thus, although we cannot compare the sensitivity of ESPVR with changes in contractility relative to other indices, it does change as expected, showing a limited response to $\beta$-adrenergic stimulation, but a large response to $\beta$-adrenergic inhibition.

It is important to note that, although our preparation is stable with respect to ESPVR over time, there is a large amount of interanimal variability. This variability probably stems from many causes, including differences in $\alpha$, geometrical and size differences among hearts, different baseline cardiorespiratory status, etc. Whatever the mechanisms for this variability, it can be taken into account by using dummy variables in multiple regression analyses or separately evaluating interanimal differences in analysis of variance models. It is clear that the ESPVR cannot be used to obtain indices such as $E_{\text {es }}$ and $V_{14}$ to yield absolute values of contractility that are comparable between animals.

In summary, we have found that the ESPVR can be reliably and reproducibly obtained in the newborn lamb using instantaneous conductance measurements of volume and micromanometric measurements of pressure by transiently occluding either the IVC or aorta, but not by volume infusion. Nonlinearity of the ESPVR is present, but not large in the range of measurement, particularly during IVC occlusion. However, extrapolation of the ESPVR well beyond the range of measurement, as is done in the calculation of $V_{d}$, is of questionable validity. The ESPVR changes only minimally in response to $\beta$-adrenergic stimulation, but changes to a great extent in response to $\beta$-adrenergic inhibition, confirming previous studies. It appears that the conductance technique for instantaneous evaluation of cardiac function in the PV plane is feasible in the newborn, and may yield new information on the normal development of the heart in situ and its response to various pathophysiologic stresses.

\section{REFERENCES}

1. Rudolph AM, Heymann MA 1970 Circulatory changes during growth in the fetal lamb. Circ Res 26:289-299

2. Teitel DF, Iwamoto HS, Rudolph AM 1987 Effects of birth-related events on central blood flow patterns. Pediatr Res 22:557-566

3. Anderson DF, Bissonnette JM, Faber JJ, Thornburg KL 1981 Central shun flows and pressures in the mature fetal lamb. Am J Physiol 241:H60-H66

4. Lister G, Walter TK, Versmold HT, Dallman PR, Rudolph AM 1979 Oxygen delivery in lambs: cardiovascular and hematologic development. Am I Physiol 237:H668-H675

5. Teitel DF, Sidi D, Chin T, Brett C, Heymann MA, Rudolph AM 1985 Developmental changes in myocardial contractile reserve in the lamb. Pediatr Res 19:948-955

6. Riemenschneider TA, Brenner RA, Mason DA 1981 Maturational changes in myocardial contractile state of newborn lambs. Pediatr Res 15:349-356

7. Berman Jr W, Musselman J 1979 Myocardial performance in the newborn lamb. Am J Physiol 237:H66-H70

8. Maylie JG, Thornburg KL, Faber JJ 1978 Force-frequency relations of the neonatal cat heart. In: Longo LD, Reneau DD (eds) Circulation in the Fetus and Newborn. Garland, New York, pp 391-398

9. Maylie JG 1982 Excitation-contraction coupling in neonatal and adult myocardium of cat. Am J Physiol 242:H834-H843

10. Nakanishi T, Okuda H, Kamata K, Abe A, Sekiguchi M, Takao A 1987 Development of myocardial contractile system in the fetal rabbit. Pediat Res 22:201-207

11. Van den Bos GC, Elzinga G, Westerhof N, Noble MIM 1973 Problems in the use of indices of myocardial contractility. Cardiovasc Res 7:834-848

12. Elzinga G, Westerhof $\mathrm{N} 1979$ How to quantify pump function of the heart. Circ Res 44:303-308

13. Baan J, van der Velde ET 1988 Sensitivity of left ventricular end-systolic pressure-volume relation to type of loading intervention in dogs. Circ Res 62:1247-1258

14. Kass DA, Maughan WL, Guo ZM, Kono A, Sunagawa K, Sagawa K 1987 Comparative influence of load versus inotropic states on indexes of ventricular contractility: experimental and theoretical analysis based on pressurevolume relationships. Circulation 76:1422-1436

15. Little WC, Cheng CP, Peterson T, Vinten JJ 1988 Response of the left ventricular end-systolic pressure-volume relation in conscious dogs to a wide range of contractile states [published erratum appears in Circulation 79:205, 1989]. Circulation 78:736-745

16. Maughan WL, Sunagawa K, Burkhoff D, Graves WL, Hunter WC, Sagawa K 1985 Effect of heart rate on the canine end-systolic pressure-volume relationship. Circulation 72:654-659

17. Mehmel HC, Stockins B, Ruffmannn K, von Olshausen K, Schuler G, Küble W 1981 The linearity of the end-systolic pressure-volume relationship in man and its sensitivity for assessment of left ventricular function. Circulation 63:1216-1222

18. Sagawa K 1978 The ventricular pressure-volume diagram revisited. Circ Res 43:677-687

19. Suga H, Sagawa K 1974 Instantaneous pressure-volume relationships and their ratio in the excised, supported canine left ventricle. Circ Res 35:117-126

20. Mirsky I, Tajimi T, Peterson KL 1987 The development of the entire systolic pressure-volume and ejection fraction-afterload relations: a new concept of systolic myocardial stiffness. Circulation 76:343-356

21. McKay RG, Spears JR, Aroesty JM, Baim DS, Royal HD, Heller GV, Lincoin W, Salo RW, Braunwald E, Grossman W 1984 Instantaneous measurement of left and right ventricular stroke volume and pressure-volume relationships with an impedance catheter. Circulation 69:703-710

22. McKay RG, Aroesty JM, Heller GV, Royal HD, Warren SE, Grossman W 1986 Assessment of the end-systolic pressure-volume relationship in human beings with the use of a time-varying elastance model. Circulation 74:97104

23. Sodums MT, Badke FR, Starling MR, Little WC, O'Rourke RA 1984 Evaluation of left ventricular contractile performance utilizing end-systolic pressure-volume relationships in conscious dogs. Circ Res 54.731-739

24. Gupta KB, Bavaria JE, Ratcliffe MB, Edmunds LJ, Bogen DK 1989 Measurement of end-systolic pressure-volume relations by intra-aortic balloon occlusion. Circulation 80:1016-1028

25. Burkhoff D, Sugiura S, Yue DT, Sagawa K 1987 Contractility-dependent curvilinearity of end-systolic pressure-volume relations. Am J Physiol H1218-H1227

26. Kass DA, Beyar R, Lankford E, Heard M, Maughan WM, Sagawa K 1989 Influence of contractile state on curvilinearity of in situ end-systolic pressurevolume relations. Circulation 79:167-178.

27. Su JB, Crozatier B 1989 Preload-induced curvilinearity of left ventricular endsystolic pressure-volume relations. Effects on derived indexes in closed-chest dogs. Circulation 79:431-440

28. Van der Velde ET 1989 Non-linearity and afterload sensitivity of the endsystolic pressure-volume relation of the canine left ventricle in vivo. In: Ventricular Pressure-Volume Relations and Loading Conditions in Vivo: Methods and Mechanisms. "All In" BV, Katwijk, The Netherlands, pp 135159

29. Baan J, Van der Velde ET 1989 Ventricular pressure-volume relations demonstrate positive inotropic effect of increased arterial impedance. In: Hori M, Suga H, Baan J, Yellin EL (eds) Cardiac Mechanics and Function in the Normal and Diseased Heart. Springer-Verlag. Tokyo, pp 189-197

30. Maughan WL, Sunagawa K, Burkhoff D, Sagawa K 1984 Effect of arterial impedance changes on the end-systolic pressure-volume relation. Circ Res 54:595-602

31. Baan J, Jong TTA, Kerkhof PLM, Moene RJ, Van Dijk AD, Van der Velde ET, Koops J 1981 Continuous stroke volume and cardiac output from intraventricular dimensions obtained with impedance catheter. Cardiovasc Res $15: 328-334$

32. Baan J, Van der Velde ET, De Bruin HG, Smeenk GJ, Koops J, Van Dijk AD, Temmerman D, Senden J, Buis B 1984 Continuous measurement of left ventricular volume in animals and humans by conductance catheter. Circulation 70:812-823

33. Baan J, van der Velde ET, van Dijk AD, Glantz SA, Diethelm L, Lipton MJ 1987 Dynamic volume of the left ventricle at 5 levels by conductance catheter and cine CT scanner. Circulation 76:IV-6

34. Burkhoff D, Van der Velde ET, Kass D, Baan J, Maughan WL, Sagawa K 1985 Accuracy of volume measurement by conductance catheter in isolated, ejecting canine hearts. Circulation 72:440-447

35. Kass D, Maughan WL 1988 From 'Emax' to pressure-volume relations: broader perspective. Circulation 77:1203-1212

36. Nozawa T, Yasumura Y, Futaki S, Tanaka N, Uenishi M, Suga H 1988 Efficiency of energy transfer fron pressure-volume area to external mechanical work increases with contractile state and decreases with afterload in the left ventricle of the anesthetized closed-chest dog. Circulation 77:1116-1124

37. Cassidy SC, Teitel DF 1990 The conductance catheter technique for measurement orf left ventricular volume in young piglets. Pediatr Res 27:16A(abstr)

38. Lew WY 1988 Time-dependent increase in left ventricular contractility following acute volume loading in the dog. Circ Res 63:635-647

39. Brace RA 1977 Fitting straight lines to experimental data. Am J Physiol 233:R94-R99

40. Boltwood Jr CM, Appleyard RF, Glantz SA 1989 Left ventricular volume measurement by conductance catheter in intact dogs: parallel conductance volume depends on left ventricular size. Circulation 80:1360-1377

41. Klopfenstein HS, Rudolph AM 1978 Postnatal changes in the circulation and responses to volume loading in sheep. Circ Res 42:839-845

42. Romero TE, Friedman WF 1979 Limited left ventricular response to volume overload in the neonatal period: a comparative study with the adult animal. Pediatr Res 13:910-915

43. Kass D, Yamazaki T, Burkhoff D, Maughan WM, Sagawa K 1986 Determi- 
nation of left ventricular end-systolic pressure-volume relationships by the conductance (volume) catheter technique. Circulation 73:586-595

44. Glower DD, Spratt JA, Snow ND, Kabas JS, Davis JW, Olsen CO, Tyson GS Sabiston Jr DC, Rankin JS 1985 Linearity of the Frank-Starling relationship in the intact heart: the concept of preload recruitable stroke work. Circulation 71:994-1009

45. Little WC 1985 The left ventricular dP/dtmax-end-diastolic volume relation in closed-chest dogs. Circ Res 56:808-815

46. van der Linden LP, van der Velde ET, Bruschke AV, Baan J 1988 Identifiability of left ventricular end-systolic pressure-volume relationships. Am J Physiol H1113-H1124

47. Suga H, Yamakoshi K 1977 Effects of stroke volume and velocity of ejection on end-systolic pressure of canine left ventricle. End-systolic volume clamping. Circ Res 40:445-450

48. Sugiura S, Hunter WC, Sagawa K 1989 Long-term versus intrabeat history of ejection as determinants of canine ventricular end-systolic pressure. Circ Res 64:255-264

49. Hunter WC 1989 End-systolic pressure as a balance between opposing effects of ejection. Circ Res 64:265-275

50. Van Hare GF, Hawkins JA, Schmidt KG, Rudolph AM 1990 The effects of increasing mean arterial pressure on left ventricular output in newborn lambs. Circ Res 67:78-83

51. Berman Jr W, Musselman J, Shortencarrier R 1980 The physiologic effects of digoxin under steady-state drug conditions in newborn and adult sheep. Circulation 62:1165-1171

52. Baylen B, Agata X, Padbury J, Emmanouilides G 1987 Adrenal catecholamine: a relationship to basal left ventricular performance and contractility in newborn lambs. Pediatr Res 21:382A(abstr)

53. Glantz SA, Slinker BK 1990 Primer of Applied Regression and Analysis of Variance. McGraw-Hill, New York

54. Slinker BK, Glantz SA 1990 Missing data in two-way analysis of variance. Am J Physiol 258:R291-R297

\section{APPENDIX}

To test for nonlinearity of the ESPVR, we considered the polynomial fit to be statistically better if both the $t$ statistic for the second-order term of the polynomial regression and the $F$ statistic comparing the two fits were significant at a $p$ value $\leq 0.01$. The $F$ statistic was calculated as:

$$
F=\frac{\text { (regression } \mathrm{SS}_{\text {poly }}-\text { regression } \mathrm{SS}_{\text {linear }} \text { ) }}{\text { residual } \mathrm{MS}_{\text {poly }}}
$$

where SS is the sum of squares, MS is the mean square, the number of degrees of freedom in the numerator equals 1 , and the number degrees of freedom in the denominator is equal to the residual degrees of freedom of the polynomial (poly) regression.

To test the sensitivity of the ESPVR to drug administration, to loading technique (comparing IVC to aortic occlusion; volume infusion was not included in this analysis because it was abandoned after the first five lambs), and to stability over time (comparing the first control run to the second control run), multiple linear regression analyses were performed with dummy variables using effects coding $(53,54)$. The regression model was

$$
\mathrm{Y}=\mathrm{b}_{0}+\sum_{i=1}^{4} \mathrm{~b}_{\mathrm{d}_{\mathrm{i}}} \mathrm{D}_{\mathrm{i}}+\mathrm{b}_{\mathrm{l}} \mathrm{L}+\mathrm{b}_{\mathrm{t}} \mathrm{T}+\sum_{j=1}^{8} \mathrm{~b}_{\mathrm{a}_{j}} \mathrm{~A}_{\mathrm{j}}
$$

where $\mathrm{Y}$ is the dependent variable $\left(\mathrm{E}_{\mathrm{es}}\right.$ or $\left.\mathrm{V}_{14}\right)$ and $\mathrm{b}_{0}$ (the intercept of the equation) is its mean value over all the runs. There were four drug variables, $D_{1}$ to $D_{4}$, representing the five drug conditions [a run during a low-level infusion of dobutamine was assigned a set of $(1,0,0,0)$; mid level $(0,1,0,0)$; high level $(0,0,1,0)$; propranolol $(0,0,0,1)$; and no drug $(-1,-1,-1,-1)]$; one loading technique variable, $\mathrm{L}$ (IVC occlusion was assigned a value of 1 and aortic occlusion, -1 ); one time lapse variable, $T$ (the 1st control period was assigned 1 , the 2 nd control -1 , and all other runs 0 ); and eight animal variables, $A_{1}$ to $A_{8}$ [the 1st lamb was assigned a set of $(1,0,0,0,0,0,0,0)$, the second $(0,1,0,0,0,0,0,0)$, etc. and the 9 th lamb was assigned a set of $(-1,-1,-1,-1,-1,-1,-1,-1,-1)]$. The coefficients (b) for each dummy variable estimate the changes, and their significance, from the overall mean value associated with that variable (for example, $b_{1}$ is an estimate of the effect of IVC occlusion on the overall mean value of the dependent variable; the difference between mean values of aortic and IVC occlusion is estimated by doubling $\left.b_{1}\right)$. Interactive effects between the drug variables and the type of occlusion were considered (as separate variables $\mathrm{D}_{1} \cdot \mathrm{L}$, etc.), by adding them to the equation individually and together. However, not one of these interactive variables significantly affected the regression equation, so they were removed from the equation. To determine the statistical significance of any variable or set of variables, an $F$ test was performed by dividing the mean square of that variable or set of variables by the mean square of the residual error. For individual variables, the mean square is equal to the type III sum of squares of that variable (the number of degrees of freedom for each variable in a linear regression model being 1). For a set of variables, the mean square was calculated by summing the type III sum of squares and dividing by the number of variables. As an estimate of between-lamb variability, the SD of the eight lamb coefficients $\left(b_{\alpha_{1}}\right.$ to $\left.b_{\alpha_{8}}\right)$, was calculated.

Using this technique of multiple regression analysis with dummy variables, it is important to realize that differences between lambs in absolute volumes may exist because of differences in the slope constant $\alpha$ and geometric differences that affect the reliability of the formula that converts segmental conductances to volumes, and that these differences are considered in the dummy variables that represent interanimal variability. Thus, absolute values of the descriptors of the ESPVR, $V_{14}$ and $E_{e s}$, cannot be compared between lambs; only the effects of various interventions on the ESPVR can be meaningfully analyzed. Whatever the technique of data analysis, it is important to realize that all techniques that estimate ventricular volume according to some formula (e.g. echocardiography, sonomicrometry, and cineangiography) will be affected by differences between animals, and thus comparisons of absolute values of any variable or index of ventricular function should not be undertaken. 